\title{
El concepto de pre-mapa como herramienta para abordar el análisis espacial
}

\section{The pre map as a tool to address spatial analysis}

\author{
Nelcy Azucena Hernández*
}

\section{Resumen}

El contenido de este artículo presenta los resultados de la investigación sobre modelos, con énfasis en aquellos que permiten la representación gráfica de variables, que intervienen en la ocurrencia de un fenómeno, sobre lo cual se desarrolla el concepto de premapa, como elaboración conceptual de relaciones espaciales entre dichas variables y como instrumento de análisis y representación, previa elaboración de un mapa analítico o sintético. Todo esto, teniendo como referencia el concepto de espacio como objeto de estudio de la geografía, para responder a una necesidad dentro del proceso de investigación geográfica, en donde aún no se elabora cartografía temática a partir de planteamientos conceptuales con ayuda de premapas y, por lo tanto, no se reconoce su valor como elementos necesarios en el correcto tratamiento dela información espacial.

Palabras clave: Análisis espacial; Cartografía Temática; M odelo Gráfico; Premapa; Síntesis Espacial. 


\section{Abstract}

This paper presents the results on model research with emphasis on those models that enable the graphic representation of variables. Those variables intervene in the occurrence of a phenomenon, over which the concept of a preamp is developed, including the conceptual elaboration of spatial relations among variables and as an instrument of analysis and representation. Prior to this is the elaboration of an analytical or synthetic map. All of the above having as a reference the concept of space as an object of study in geography. The purpose is to answer a need within the geographical research process, when thematic cartography is not yet elaborated, based on the conceptual formulation with the aid of premaps. As such, their value as necessary elements in the correct treatment of spatial information y yet to be acknowledged.

Key words: Graphic Model, Premap; Spatial analysis, Thematic Cartography. 


\section{Introducción}

Dentro del espacio geográfico se encuentra distribuida una serie de elementos relacionados entre sí, que son objeto de análisis para el geógrafo y por lo cual éste, constantemente, se halla ante el problema que plantea su manejo. A su vez, es bien conocida la importancia de los mapas como modelos de representación del mundo real y, por lo tanto, de organización espacial. Cada una de las realidades objeto de análisis, constituye un grado de complejidad acorde con la cantidad de información extractada, ligada al patrón de organización del fenómeno analizado y que necesita ciertas condiciones para que, al ser mostrada, exprese claramente aquello que se quiere dar a conocer.

Ahora bien, en geografía es normal hablar de la elaboración de mapas, como representaciones gráficas constituidas en fuente de información, en las que se pueden encontrar localizados los elementos visibles del paisaje, sobre los que se toman medidas o los que exhiben, mediante el uso de símbolos de carácter cualitativo o cuantitativo, una repre-sentación convencional de fenómenos localizables y de sus relaciones (Board, 1967); sin embargo, en el espacio geográfico, están presentes unas estructuras y unas funciones que deben ser consideradas de manera holística, antes de empezar el proceso cartográfico. El reconocimiento de estas propiedades implica el estudio de las relaciones espaciales, mediante el uso de modelos que representen su distribución y organización.

Como lo muestra la Figura 1, tradicionalmente, a partir de la distribución de un fenómeno objeto de estudio y con base en procedimientos apropiados para la toma de datos, se hace una representación cartográfica, cuya función es registrar y comunicar las observaciones del investigador, en forma de lenguaje gráfico; es decir, se pasa de los datos organizados respondiendo a un orden gráfico, al mapa de manera directa, sin pasos analíticos intermedios, que permitan integrar la información generada a partir de los datos.

Para entender fácilmente el comportamiento de dicho fenómeno, se debe recurrir a los modelos que están pensados para ayudar a visualizar y comprender su complejidad, transformándolo a un sistema simple que facilite su interpretación (Harvey, 1983). 


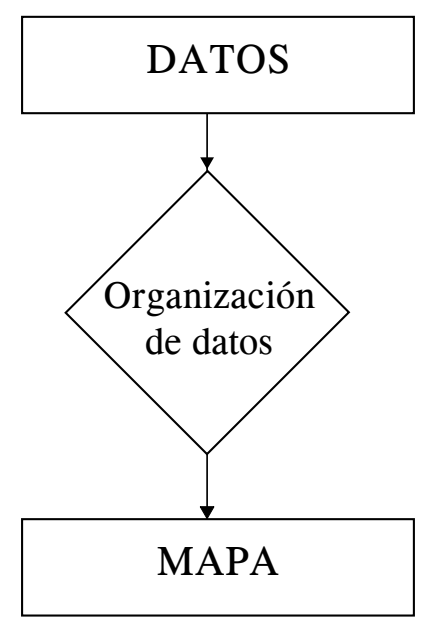

Figura 1. Proceso tradicional de elaboración de un mapa

Las representaciones cartográficas así concebidas, aunque cumplen en gran medida con su objetivo de mostrar un resultado en algunas ocasiones confuso, tienden a alejarse de lo que sería su correcta elaboración temática, puesto que, para ello, han debido hacer uso de elementos de análisis sin conceptualizar, pero que no por ello han dejado de existir; es decir, elementos que no han sido definidos $y$, por lo tanto, tampoco aprovechados, pero que han existido de manera anónima y han sido utilizados sin un marco conceptual que los contenga.

Algunos autores han reconocido el potencial de los premapas y los han mencionado, sin llegar todavía a profundizar al respecto. En Bunge (1967), trabajo consultado sobre el desarrollo de la metacartografía, se menciona la existencia de los premapas con un enfoque diferente al que aquí se plantea; allí, se trata de encontrar el límite entre ellos y los mapas, abordando el estudio de propiedades, como escala, forma y distorsión entre otras.

\section{M etodología}

La investigación presentada aquí es de carácter exploratorio y documental, por lo cual tiene la capacidad de ofrecer elementos de análisis, surgidos a partir de los procesos llevados a cabo durante el abordaje del estudio del concepto de PREMAPA, teniendo como base la teoría espacial en el marco de la geografía y haciendo referencia al concepto de espacio relativo, como el principal objeto de análisis y representación.

Se planteó la investigación desde tres líneas básicas de exploración: la primera, relacionada con el manejo conceptual de la taxonomía, para orientar la clasificación propuesta, 
siguiendo los principios básicos del establecimiento de tipologías planteados por Hempel (1988, 1965), Nagel (1972, 1968), Zonneveld (1988), Buzai (2003) y Moreno (1994). La segunda, relacionada con la teoría general de modelos, desde el momento en que son concebidos, lo cual sirve para lograr el entendimiento del proceso lógico que se necesita para representar la realidad, acudiendo aquí a autores como Chorley y Haggett (1967), Krugman (1991), Minshull (1975), Ferras (1993), Richards (2004) ý Bord (1984), entre otros. Y en tercer lugar, la relacionada directamente con los modelos gráficos llamados premapas, para lo cual se ha hecho la revisión de documentos gráficos en atlas de IGAC (1967, 1969, 1977, 1992, 2002), IDEAM (2001), INGEOMINAS (2005), entre otros, en donde se pueda estudiar la manera como se maneja la información espacial y la utilización de los conceptos que permiten hacer la representación gráfica o cartográfica de los fenómenos.

Si bien es cierto que los modelos son vistos como representaciones que permiten dar a conocer la realidad de manera simplificada, también es cierto que existen elementos que, siendo modelos, conscientemente no han sido reconocidos como tal, aunque se utilicen a diario. Dada tal circunstancia, se tratará aquí de recogerlos y agruparlos de la manera natural como son percibidos y utilizados, de tal forma que este se convierte en un ejercicio de observación y clasificación, que permitirá llegar hasta el límite en donde se insertan los premapas como modelos gráficos.

\section{Resultados y Discusión}

\subsection{E I concepto de M odelo}

Los modelos ayudan a conocer el comportamiento de los sistemas (Bailly, 1978, citado por Rodríguez, 2000); por lo tanto, se constituyen en una manera práctica de abordar el análisis espacial, sobre las categorías fundamentales del conocimiento geográfico, como el espacio, el lugar, la región, el territorio, el hábitat y el paisaje, para citar sólo algunas, que permiten definir el objeto de estudio de la geografía; así, se convierten en una herramienta útil con la cual abordar el análisis de estructura $\mathrm{y}$ funcionamiento de la realidad.

Estos términos mencionados forman parte de la estructura del espacio, en su carácter de realidad relacional; es decir, en su calidad de espacio relativo, que existe sólo por la presencia de unos objetos que están relacionados entre sí y que mantienen una dinámica propia (Mabogunje, 1980, citado por Santos, 1996) y que, por lo tanto, son objeto de representación con base en los modelos espaciales, que permiten al geógrafo hablar a través de las barreras del lenguaje (Sauer, 1956, citado por Córdoba y Ordóñez, 2004).

El diseño de modelos forma parte del proceso de análisis de la realidad; por lo tanto, se puede pensar en ellos como una forma de abstracción, que permite 
descomponer conceptualmente el espacio geográfico para entender su complejidad, a través del conocimiento de una parte finita, dentro de la infinita multitud de fenómenos que lo componen (Weber citado por Rodríguez, 2000).

El aspecto más importante aquí es el estudio de las relaciones espaciales; por lo tanto, al consultar diferentes textos sobre modelos, se observa que, científicamente, su existencia tiene origen en la apreciable necesidad del hombre por entender su variedad de relaciones con el entorno, así como de explicar su comportamiento (Jeffers, 1991). Es decir, son una reacción humana ante la complejidad del mundo que le rodea, los cuales le permiten elaborar una visión simplificada de la realidad (Chorley y Haggett, 1967); los autores lo llaman "necesidad de idealización". Sin embargo, pueden considerarse como sistemas definibles, con base en el interés de quien los construye, para responder preguntas relacionadas con la organización espacial, a partir de diferentes variables, que es uno de los intereses de la geografía.

Un modelo es entonces una estructuración simplificada de la realidad, que presenta, de forma generalizada, sus elementos significativos e ignora las especificidades que se consideran ruido, dentro del sistema que se genera, convirtiéndose así en una aproximación subjetiva al descartar información incidental (Chorley y Haggett, 1967). Es, por lo tanto, una idealización de la realidad, utilizada para plantear un problema, normalmente de manera simplificada en términos relativos, y planteada desde un punto de vista matemático, y/o físico. Permite representar un conjunto de relaciones entre dos o más variables, con el fin de estudiar los efectos provocados por la variación de una de ellas sobre las demás, lo cual le da una propiedad para ser tenido en cuenta como premapa, en el análisis del espacio geográfico.

En toda investigación, y en particular en geografía, los modelos facilitan el conocimiento de los niveles de integración entre variables, de distribución espacial, de su estructura y de sus funciones, para permitir la comprensión de un fenómeno a través del acercamiento detallado a sus elementos constitutivos y a sus relaciones; en general, se habla de ellos como una representación abstracta de una realidad concreta (Durand, 1979).

\subsection{Clasificación general de M odelos}

Como resultado del ejercicio de observación de los modelos existentes, se llega a determinar que la clasificación de modelos apropiada para el tema de investigación, debe tener como criterios, en primer lugar, el orden en que son concebidos en el cerebro del ser humano, lo cual facilitará la elección de su forma de expresión y de representación; y en segundo lugar, para el siguiente nivel, la utilidad que pueda hacerse de cada elemento. 
Atendiendo la primera parte, se observa en la Figura 2 cómo es la clasificación de modelos desde su proceso de concepción hasta su materialización. Allí, los números señalan el orden en que empiezan a tener existencia los modelos, empezando en el 1 con los mentales, hasta llegar al 5, cuando son plasmados de manera física o materializados. La forma como se relaciona un modelo mental con uno gráfico, puede ser un premapa, si existe una variable espacial que ayude a configurar el modelo gráfico y represente las posibles relaciones entre los objetos antes de construir el propio modelo.

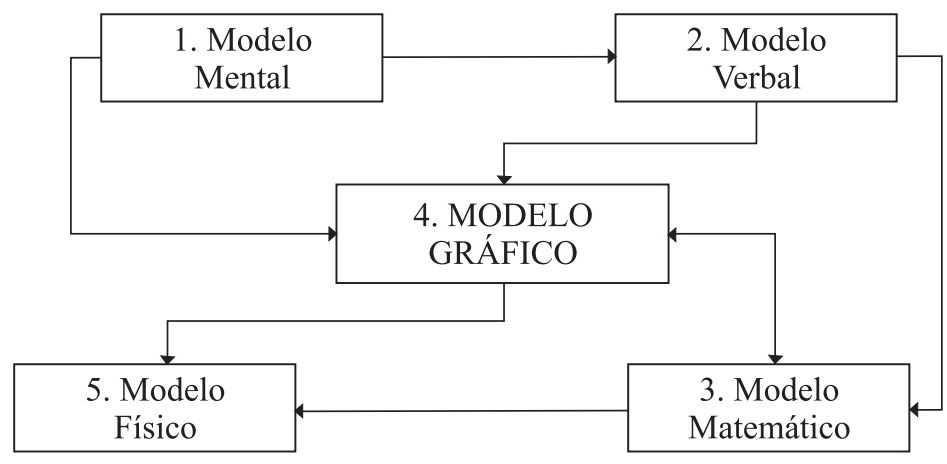

Figura 2. Clasificación general de modelos a partir de su proceso de concepción hasta su materialización

El gran universo lo constituye el conjunto de todos los modelos existentes, como se ve en la Tabla 1, en donde se resume la clasificación encontrada. Dentro de dichos modelos, existe una serie de divisiones a las que se llamará clases, establecidas según el orden que sigue el proceso de su concepción y representación; estas clases se mencionan en el orden como se presentan ante la percepción humana, y obedecen a su forma de expresión. Una vez establecida esta clasificación general, se encontró que, según su utilidad, al interior de cada una de ellas, existen otras divisiones que se denominarán, en su orden, subclase y género, en donde están ubicados los premapas dentro de los modelos gráficos. 
Tabla 1. Resumen de la clasificación general de modelos

\begin{tabular}{|c|c|c|c|}
\hline CLASE & SUBCLASE & GÉNERO & EJEMPLO \\
\hline \multirow[t]{4}{*}{ MENTALES } & \multirow{2}{*}{ CREATIVOS } & & Ideas \\
\hline & & & Objetos mentales \\
\hline & \multirow[t]{2}{*}{ INDICATIVOS } & & Señales de tránsito \\
\hline & & & Señales de seguridad \\
\hline \multirow[t]{4}{*}{ VERBALES } & \multirow[t]{2}{*}{ NORMATIVOS } & & Metadiscursos \\
\hline & & & Metodologías \\
\hline & \multirow[t]{2}{*}{ INVESTIGATIVOS } & & $\begin{array}{l}\text { Teoría del origen del } \\
\text { Universo }\end{array}$ \\
\hline & & & Teoría genética \\
\hline \multirow[t]{2}{*}{ MATEMÁTICOS } & DESCRIPTIVOS & & Ley de Newton \\
\hline & PREDICTIVOS & & Modelos de regresión \\
\hline \multirow[t]{9}{*}{ GRÁFICOS } & \multirow[t]{2}{*}{ SIMBÓLICOS } & ARTÍSTICOS & Caricaturas \\
\hline & & ICÓNICOS & Fotografía \\
\hline & \multirow[t]{4}{*}{ FUNCIONALES } & \multirow{3}{*}{\begin{tabular}{|l|} 
CÍCLICOS \\
DE PROCESOS
\end{tabular}} & Ciclo del agua \\
\hline & & & Ciclo del carbono \\
\hline & & & Modelo cartográfico \\
\hline & & ORGANIZACIONALES & Christaller, Von Thünen \\
\hline & \multirow[t]{3}{*}{ GEO-GRÁFICOS } & PREMAPAS & Diagrama de barras \\
\hline & & & Diagrama Triangular \\
\hline & & MAPAS & Temáticos \\
\hline \multirow[t]{2}{*}{ FISICOS } & DIDACTICOS & & Globo terráqueo \\
\hline & EXPERIMENTALES & & Modelo de una presa \\
\hline
\end{tabular}

3.3 M odelos gráficos como Premapas

Efectuada una revisión de bibliografía en trabajos de grado, textos y revistas de corte geográfico, se encontró una escasa producción sobre los premapas, aunque en realidad, su uso aparece con relativa frecuencia a manera de discretos gráficos acompañando un texto o describiendo un comportamiento de variables, pero nunca como premapa; es decir, como paso previo a la obtención de un mapa, lo cual implica una elaboración conceptual de las relaciones espaciales entre las variables que intervienen en el fenómeno estudiado; aspecto que es importante resaltar aquí y que implica el estudio de una serie de procesos lógicos que hay que reconocer.

Bunge (1967), propone llamar premapas a las fotografías aéreas o terrestres, los resultados de encuestas o de censos, las definiciones de límites administrativos, los registros de aparatos meteorológicos, entre otros, y separarlos de los mapas, así como de las matemáticas aplicadas a ellos, los cuales introducen la información en sistemas lógicos para ser tratados. Un premapa, en estas condiciones, es un instrumento que carece de propiedades racionales para mostrar fenómenos espaciales, tales como escala, proyección, generalización y/o 
leyenda; es simplemente un elemento previo informativo antes del mapa, utilizado en la primera etapa de la investigación geográfica: la recolección de datos (Rimbert, 1968).

Lo anterior muestra los premapas como herramientas base que permiten o facilitan la descripción; sin embargo, en el presente documento se estudian, en su connotación, como instrumentos de análisis, previa elaboración de un producto cartográfico, mediante el establecimiento de las relaciones entre variables, para lo cual, el paso siguiente, será profundizar en su conocimiento y en sus potencialidades, y seleccionar, de entre la gama de diagramas existentes para representar información espacial, aquellos que permiten llegar al mapa.

En 1991 aparece un artículo sobre la información geo-gráfica, que enfatiza en los diagramas; en donde se afirma que éstos adquieren el carácter de premapa, en cuanto describen la distribución espacial de un fenómeno y, por ende, puede ser plasmada en un fondo cartográfico (Caycedo y Flórez, 1991), lo cual lleva a pensar que un premapa, como su nombre lo indica, no sólo antecede a un mapa, sino que es una condición necesaria para que éste último exista.

Un premapa puede hablar por sí mismo, y talvez no sea necesario configurar un mapa como paso posterior, si es que existen ciertas propiedades que se verán más adelante. Este se constituye en un primer acercamiento a estos instrumentos de análisis, dentro del proceso de investigación en geografía, haciendo un intento por mostrar su utilidad como elaboración conceptual de relaciones cuantitativas o cualitativas, que muestra parte de la organización del espacio, en términos ya sea estructurales o funcionales, lo cual induce a pensar en su papel como instrumento de apoyo para llegar al mapa, en tanto muestra distribuciones espaciales.

En el año 2005 se publicó un libro originado de una investigación efectuada en el Departamento de Geografía de la Universidad Nacional, en donde sus autoras definen el premapa como un mapa en potencia, cuya importancia se centra en su capacidad para establecer y/o mostrar relaciones (Madrid y Ortíz, 2005). Al manejarlo en la construcción de mapas de isolíneas sugieren, como proceso previo, el conocimiento de la variación de un elemento en relación con otro, mediante el establecimiento de una correlación entre variables. Estos antecedentes en conjunto, constituyen la base sobre la cual se intentará construir la teoría de los premapas, que forman parte de una serie lógica de etapas para hacer más comprensibles las relaciones espaciales.

Tomando como referencia el caso de la construcción de isopletas, las etapas mencionadas inician con la elaboración de gráficas calibradoras, que caractericen la distribución espacial de las variables estudiadas, pasando por el diseño de diagramas de dispersión, que muestren cuál es la relación entre esas variables, hasta llegar a los mapas de 
isolíneas; es decir, no es simplemente tomar datos y generar líneas o áreas de valores similares a partir de una interpolación; es llegar a comprender el fenómeno, para que pueda ser representado por medio de un modelo gráfico, como el mapa, tomando como punto de partida el estudio de las relaciones entre variables, con ayuda de elementos gráficos como los premapas.

Esta idea constituye una propuesta inicial para empezar a conceptualizar los premapas, reconocidos como modelos gráficos en sus inicios, y desde ahí encontrar elementos que apoyen su construcción, a partir de la información que se tenga, en la búsqueda de un objetivo común, que es una representación cartográfica final. En definitiva, la base de este ejercicio investigativo se encuentra en los textos mencionados, de los cuales se tomaron algunos elementos para fortalecer las ideas que aquí se proponen, así como se descartó otros, hasta lograr el propósito trazado inicialmente.

\section{4 ¿Para qué los Prempas?}

Dentro de la gran gama de hechos geográficos, se presenta una serie de correlaciones, entre las que no existe un determinismo absoluto, pero que es necesario conocer y manejar, lo cual constituye la esencia del trabajo del geógrafo (Joly, 1982), quien se ve enfrentado a proyectos en los cuales es necesario el uso de los métodos cuantitativos y cualitativos para favorecer el manejo de la información, hacer análisis y presentar resultados que ofrezcan una solución al problema que se le plantee.

En todo informe de trabajo práctico, en casi todas las áreas del conocimiento, se encuentran gráficos de diverso tipo, que ilustran y ayudan en la comprensión del problema estudiado, aunque algunas veces no se consigue este objetivo, pues se tiene una idea errónea del concepto que se debe mostrar, en el momento de hacer comprensible el texto del informe, dándole prioridad al detalle estético por encima del contenido. Otras veces se elaboran mapas, pretendiendo que éstos siempre son la mejor herramienta gráfica para mostrar el resultado de un análisis espacial, cuando en realidad, en determinadas ocasiones, son más útiles los gráficos que describen las relaciones que se están estudiando y que permiten una mejor comprensión de las estructuras espaciales.

Esto sucede porque no se hace un análisis espacial a partir de un punto inicial, que es tener claro el proceso de investigación geográfica, sin pasar por alto algún paso que resulte clave, como la confección de premapas. No significa esto que todos los trabajos elaborados presenten deficiencias desde el punto de vista gráfico, pero aparecen con frecuencia; el conocimiento de la existencia y operación de los premapas, con el enfoque que aquí se le dará, permitirá subsanar algunas de esas debilidades, teniendo presente que para analizar el espacio se necesita realizar dos etapas: la primera, descubrir los elementos 
involucrados que presentan caracteres comunes y la segunda, encontrar las relaciones que presentan esos elementos entre sí (Aguirre, 1979), de tal manera que se logre un estudio dinámico de los componentes de un medio que puede parecer estático. De esta manera se logrará saber exactamente qué es lo que se debe mostrar como resultado gráfico.

\subsection{La matriz de datos geofráfica o la base de datos relacional: el origen de todo Premapa}

Con ayuda de un ejercicio de observación y análisis de ejemplos, se encontró la matriz geográfica o base de datos relacional georreferenciada, como factor común en la elaboración de productos cartográficos dentro del proceso de investigación en geografía. Así pues, se puede afirmar, a partir de ello y de la propuesta hecha por Berry (1964) y citada por Harvey (1983), de una disposición de datos en filas (para localizar la variable espacial) y columnas (para ubicar los atributos), que la información objeto de representación debe estar organizada conceptualmente, de tal manera que se facilite su manejo. Con los avances tecnológicos esta condición no sólo sigue vigente, sino que se fortalece con la aparición de los sistemas de información geográfica (SIG), que disponen de capacidad suficiente para procesar grandes volúmenes de datos, en donde la primera operación que se realiza es su almacenamiento en una base de datos, que permita su manipulación y, si es necesario, la generación de productos cartográficos (Bolaños, 2004).
La matriz de datos geográfica implica una definición de conceptos y de escalas de medición, de acuerdo con el tipo de información que se investiga, la cual, una vez recopilada y organizada, se puede llevar a una base de datos, cuya construcción debió ser objeto del mismo tratamiento; recurrir a este elemento puede facilitar la representación y análisis, sobre todo si la cantidad de información así lo exige.

$\mathrm{Al}$ existir la posibilidad de ir de la matriz a la base de datos, existe también la misma posibilidad de considerarlas homólogas, ya que poseen características estructurales y funcionales similares; es decir, no sólo se puede afirmar que el origen de todo premapa está en la matriz de datos geográfica, sino también en la base de datos, y la elección de una u otra dependerá de la complejidad y cantidad de información presente. Al hacer referencia aquí a una u otra como origen del premapa, éstas se deben interpretar como expresiones sinónimas, con la capacidad de recolección y organización de la información y para su manipulación y análisis.

Al revisar la información de investigaciones publicadas en el país, se encuentra que ésta se tiene organizada en una base de datos que contiene la variable espacial $\mathrm{y}$, por lo tanto, es posible repre-sentarla cartográficamente. Al estudiar los distintos modelos gráficos con los que se puede tratar esta información, se aprecia que hay un elemento común a todos, que es una base de datos, tal como lo afirma Bertin (1977), como fuente de 
información espacial y, así mismo, como materia prima para elaborar gráficos, que permitan llevar a cabo el análisis del fenómeno y representarlo con la ayuda de premapas.

Ahora, una matriz o base de datos, desde este punto de vista, se compone de dos tipos de datos: el primero corresponde a la información de localización del fenómeno estudiado y el segundo a los netamente relacionados con su distribución. En definitiva es, dicha base, el primer componente de la serie lógica de etapas que conforman el proceso de investigación en geografía.
La Figura 3, muestra cómo la base de datos relacional es el origen de todo premapa, al ser el primer objeto de tratamiento de la información, puesto que es allí donde se facilita la organización y el manejo de las variables involucradas, que permitirán llegar a la representación cartográfica o decidir presentar el premapa como resultado. Así pues, si la base de datos es origen de los premapas, lo es también de los mapas, tanto cualitativos como cuantitativos, entendidos como modelos gráficos, usados para representar la organización espacial de fenómenos de la realidad, sobre temas de diversa índole, ya sean físicos, sociales, económicos o ambientales.

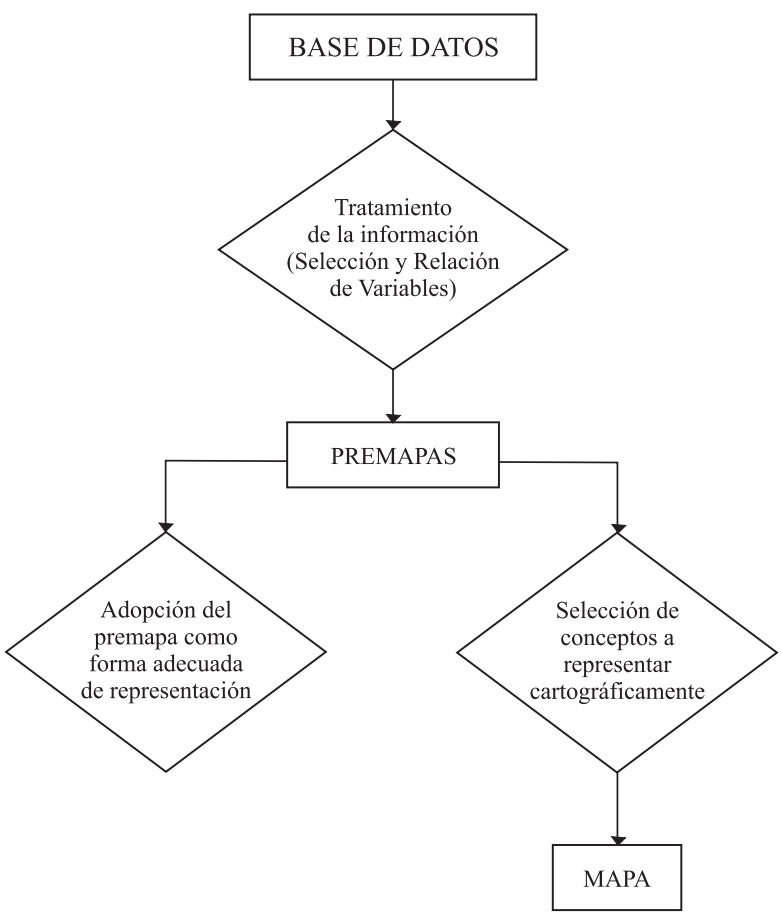

Figura 3. La base de datos relacional como origen de los premapas y mapas 
3.6 ¿C uáles son los M odelos $\mathrm{G}$ ráficos que pueden ser considerados Premapas?

Existen premapas, tanto cualitativos como cuantitativos, que pueden ser considerados premapas, aunque los primeros aparezcan con poca frecuencia en las fuentes investigadas; sin embargo, vale la pena incluirlos como una tipología dentro de la clasificación que se está planteando, que permita rescatar la representación de las relaciones cualitativas que pueden dar origen a un mapa. La Figura 4 corresponde a un diagrama de barras, en el cual se representa la variable cuantitativa nivel de fecundidad y actúa como variable espacial el conjunto de los cinco continentes; esta información puede ser llevada directamente a un mapa de la superficie terrestre, en donde se representen los continentes de forma diferencial, con ayuda de las variables visuales color, forma u orientación.

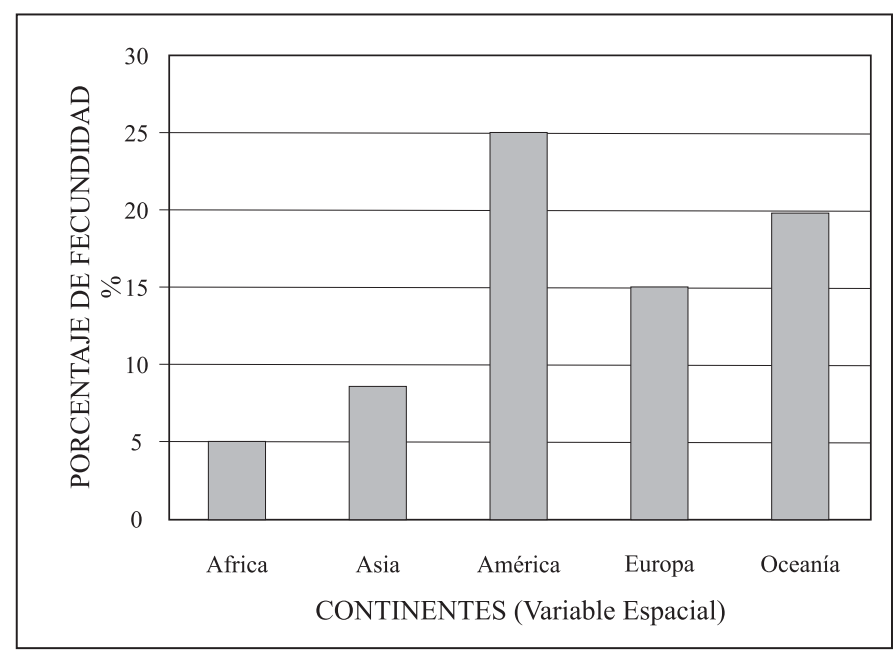

Figura 4. Premapa que muestra el nivel de fecundidad por continente 
Este premapa representa claramente la información que se necesita mostrar y, probablemente, no sea necesario recurrir a un mapa para darla a conocer; inclusive, es posible ordenar las barras en orden ascendente o descendente, de tal manera que las respuestas al lector sean más evidentes $\mathrm{y}$, por lo tanto, más rápidas.

Si se decide implantar esta información en un mapa, su lectura será similar a la mostrada en la Figura 4, la cual puede ser modificada y en donde se facilita la rapidez en las comparaciones, que serán de manera directa entre los valores que toma la variable en cada unidad espacial; en cualquier caso, el nivel de lectura aquí es elemental. Estos gráficos son muy comunes y aparecen con relativa frecuencia en los textos de geografía, así como en publicaciones de todo tipo, inclusive en las de índole geográfica; la presencia de la variable espacial hace posible clasificarlos como premapas.

En la Tabla 2, se presenta la relación de los tipos de modelos gráficos que pueden ser considerados premapas, junto con sus propiedades, producto de la observación de muchos ejemplos en donde se han utilizado, ya sea para mostrar una distribución o algún tipo de relación entre variables.

Tabla 2. Relación de modelos gráficos con carácter de premapa y sus propiedades

\begin{tabular}{|c|c|}
\hline PREMAPAS & PROPIEDADES \\
\hline $\begin{array}{l}\text { - Diagrama de barras sencillo } \\
\text { - Diagrama de barras compuesto } \\
\text { - Diagrama de sectores } \\
\text { - Diagrama de dispersión x,y } \\
\text { - Diagrama en x,y } \\
\text { - Triángulo } \\
\text { - Cuadrado } \\
\text { - Diagrama Polar } \\
\text { - Semivariograma } \\
\text { - Pictogramas } \\
\text { - Perfiles } \\
\text { - Grilla con valores de la variable pendiente, } \\
\text { definidos sobre un mapa topográfico } \\
\text { - Calcos de interpretación de fotografías } \\
\text { aéreas o imágenes de satélite } \\
\text { - Diagramascualitativos }\end{array}$ & $\begin{array}{l}\text { 1. Tiene la capacidad de mostrar distribuciones } \\
\text { espaciales de variables. } \\
\text { 2. Puede manejar variables tanto cualitativas } \\
\text { como cuantitativas. } \\
\text { 3. Puede llegar a reemplazar el mapa. } \\
\text { 4. Puede servir de elemento calibrador de un } \\
\text { mapa. } \\
\text { 5. Permite relacionar variables. } \\
\text { 6. Ofrece la posibilidad de pasar la informa- } \\
\text { ción al mapa. }\end{array}$ \\
\hline
\end{tabular}

\begin{tabular}{l|l}
\hline 68 & N. Hernández
\end{tabular} 


\subsection{Condiciones para que un Modelo gráfico sea Premapa}

Con base en la investigación documental y acudiendo a material gráfico de investigaciones geográficas, textos de geografía, trabajos de entidades estatales e informes sobre distribuciones espaciales, consultadas en medios informativos, que tienen que ver con el manejo de información espacial, se puede determinar, a partir de su observación, análisis e interpretación, que para considerar un documento no cartográfico como premapa, debe cumplir con ciertas condiciones que son la base sobre la que se toma la decisión de la clasificación propuesta:

- Contener la variable espacial.

- Permitir el paso al mapa de manera directa o indirecta.

- Manejar variables cualitativas, cuantitativas o la combinación de las dos.

- Tener su origen en una matriz base de datos georreferenciada o ser una de ellas.

- Tener la capacidad de mostrar la distribución espacial de una o más variables.
Dentro de las formas que adopta la variable espacial, estarán:

- Si es para una variable puntual serán Coordenadas $\mathrm{x}, \mathrm{y} ; \mathrm{z} ; \mathrm{f}$, ?; h, ó valores de dos variables cuantitativas.

- Pueden ser distancias a partir de un punto.

- Si es una línea será la relación espacial entre dos variables cuantitativas.

- Si lo que se quiere representar son áreas, y se habla de un diagrama en dos dimensiones, cada división en el eje $x$ representará un municipio, una ciudad o una zona geográfica en general.

\subsection{Tipología propuesta}

Dentro de los modelos gráficos se encontró el género premapa, dentro del cual existen elementos que responden a un orden cualitativo y cuantitativo, de acuerdo con el tipo de variable que manejen. A su vez, la tipología obedece al concepto de suborden, definido desde el punto de vista de la forma de pasar del premapa al mapa, ya sea directa o indirecta, tal como lo muestra la Tabla 3.

Tabla 3. Tipología de premapas como modelos gráficos

\begin{tabular}{|c|c|c|c|c|c|}
\hline CLASE & SUBCLASE & GE NERO & ORDEN & SUBORDEN & EJEM PL O \\
\hline \multirow{2}{*}{$\begin{array}{c}\text { Modelos } \\
\text { Gráficos }\end{array}$} & $\begin{array}{c}\text { Geo- } \\
\text { Gráficos }\end{array}$ & Premapas & Cualitativo & Directo & $\begin{array}{c}\text { Diagrama combinado } \\
\text { de pisos bioclimáticos }\end{array}$ \\
\cline { 4 - 6 } & & Cuantitativo & $\begin{array}{c}\text { Directo } \\
\text { Indirecto }\end{array}$ & $\begin{array}{c}\text { Diagrama de barras } \\
\text { Diagramatriangular }\end{array}$ \\
\hline
\end{tabular}




\subsection{Propuesta de concepto de Premapa}

El principal resultado de la investigación es el concepto de premapa, como modelo gráfico útil al abordar el análisis espacial y la representación de relaciones espaciales entre variables.

Premapa es una representación o conjunto de representaciones de variables espacializadas que conforman un fenómeno de la realidad, en donde, a diferencia del mapa, la ubicación espacial está dada, si es una base de datos o matriz geográfica, por un listado de coordenadas o de lugares definiendo un espacio, o si es un modelo gráfico de $\mathrm{n}$ componentes, uno de ellos debe ser la variable espacial. Sirve para mostrar distribuciones espaciales, ya sea en forma de puntos, líneas o polígonos y constituye el paso previo al mapa, ya que actúa, en primer lugar, como instrumento de análisis y, en segundo grado, como herramienta para lograr una mayor aproximación a la realidad, en la representación cartográfica de los fenómenos espaciales.

\subsection{Aplicación del concepto de Premapa: elaboración de un Mapa de Pisos Bioclimáticos}

Con base en información del trabajo de Sáenz (2003), se puede elaborar un mapa de pisos bioclimáticos de la cuenca de las quebradas Tirque y Chinicua, ubicadas en el Municipio de Socha, Departamento de Boyacá, para lo cual se dispone, en primer lugar, de una matriz o base de datos geográfica, como se muestra en la Tabla 4, que ha sido objeto de tratamiento, luego de la investigación en campo de las variables relacionadas allí.

Tabla 4. Base de datos: Premapa Origen

\begin{tabular}{|c|c|c|c|c|c|c|c|c|}
\hline \begin{tabular}{|c}
$\mathrm{X}$ \\
(por mil)
\end{tabular} & $\begin{array}{c}\mathrm{Y} \\
\text { (por mil) }\end{array}$ & $\begin{array}{l}\text { Altura } \\
\text { (msnm) }\end{array}$ & $\begin{array}{c}\text { Temp. } \\
\mathbf{T}^{\circ} \mathrm{C}\end{array}$ & $\begin{array}{l}\text { Precip. } \\
\text { mm }\end{array}$ & $\begin{array}{c}\text { Porcentaje } \\
\text { de } \mathrm{C} \text { ar- } \\
\text { bono } \% \mathrm{C}\end{array}$ & $\begin{array}{l}\text { Densidad } \\
\text { de vivien } \\
\text { da } R \text { ural } \\
\text { Viv/K m² }\end{array}$ & $\begin{array}{l}\text { Formas de } \\
\text { ocupación } \\
\text { del espacio }\end{array}$ & $\begin{array}{c}\text { Tipo de } \\
\text { modelado } \\
\text { y relieve }\end{array}$ \\
\hline 1'152 & 1'156 & 2.200 & 15.3 & 690 & 1 & 21 & $\begin{array}{l}\text { Frutales } \\
\text { y pastos }\end{array}$ & \\
\hline 1'153 & 1'154 & 2.400 & 14.4 & 715 & 1 & 19 & \begin{tabular}{|c|} 
Cultivos \\
Mixtos \\
Extracción \\
Carbón \\
\end{tabular} & $\begin{array}{l}\text { Cañón de } \\
\text { disección }\end{array}$ \\
\hline 1'154 & 1'152 & 2.800 & 11.6 & 1.230 & 2 & 9 & \begin{tabular}{|c|} 
Extracción \\
Carbón
\end{tabular} & $\begin{array}{l}\text { Glaciar con lagu- } \\
\text { nas sedimentadas }\end{array}$ \\
\hline 1'154 & 1'149 & 3.200 & 10.4 & 1.100 & 6 & 3 & $\begin{array}{l}\text { Cultivos } \\
\text { Mixtos }\end{array}$ & $\begin{array}{l}\text { y en proceso } \\
\text { de colmatación }\end{array}$ \\
\hline 1'144 & 1'157 & 3.600 & 6.5 & 1.000 & 9.6 & 2 & $\begin{array}{l}\text { Pajonal, } \\
\text { escarpes }\end{array}$ & \\
\hline
\end{tabular}

\begin{tabular}{l|l}
\hline 70 & N. Hernández
\end{tabular} 
En segundo lugar, se elabora una serie de premapas, dependiendo de la cantidad y del tipo de variables que se investigaron en campo y que se presentan en el documento mencionado, los cuales son necesarios para estudiar su comportamiento espacial. Éstas fueron: Temperatura, Precipitación, Altitud, Porcentaje de Carbono, Ocupación del espacio y Densidad de vivienda.

La Figura 5, muestra una de las posibles relaciones que se necesita estudiar; sin embargo, también se pueden graficar las relaciones de la variable espacial contra las demás variables involucradas en la organización del espacio, hasta llegar a un premapa más complejo, como el que se muestra en la Figura 6, considerado premapa síntesis, que ha sido elaborado mediante la relación de cada variable con el espacio y con la integración de todas las variables involucradas, para estudiar su variación espacial en conjunto $\mathrm{y}$, a partir de ello, poder definir la existencia de cada piso bioclimático.

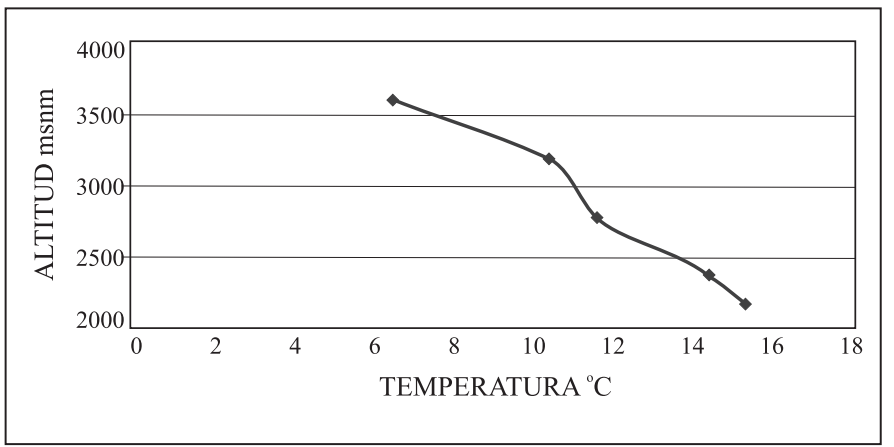

Figura 5. Relación Temperatura-Altura: Premapa Cuantitativo Directo Fuente: Sáenz, 2003. 


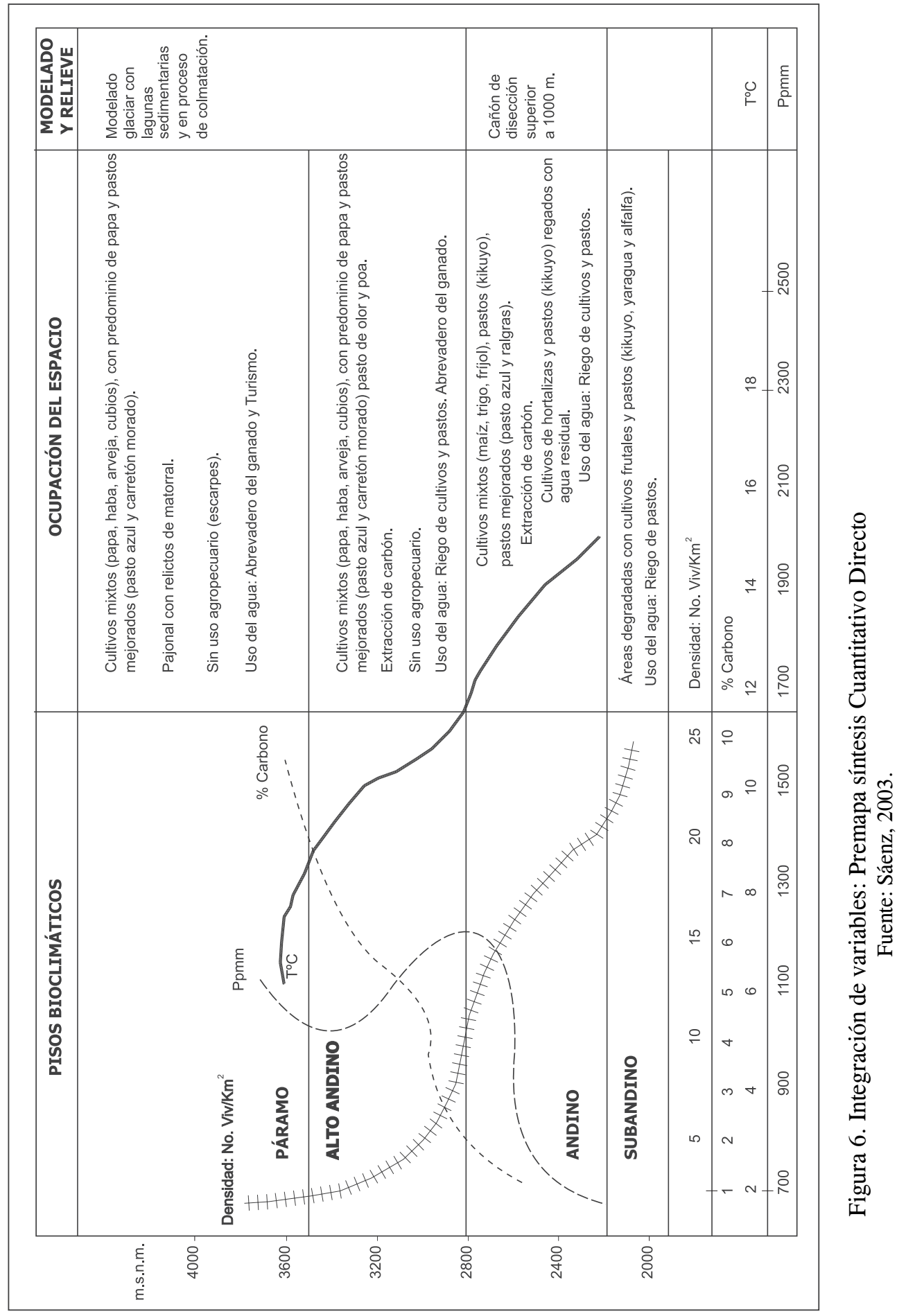


Este premapa, como síntesis espacial, tiene dos funciones: la primera, dar a conocer la dinámica del fenómeno de manera clara, a partir de la integración de elementos cualitativos; en este caso, la ocupación del espacio y el modelado, con elementos cuantitativos referidos a la medición de las variables mencionadas anteriormente. La segunda función, será permitir el paso directo a un mapa, aunque el premapa por sí solo ya muestra la distribución espacial que se puede ver en un mapa; aquí la decisión de representar la información cartográficamente, o presentar el premapa como resultado, dependerá del objetivo del investigador y del perfil de sus lectores.

El mapa de pisos bioclimáticos, conceptualizado a partir de los elementos mostrados con los premapas, constituye un concepto síntesis, al cual se llega luego de un tratamiento de la información espacial, que no se puede desconocer en el proceso de investigación, por lo cual es importante reconocer la diferencia entre la elaboración de un mapa, sin estos procesos previos, y uno a partir de ellos.

El resultado se muestra en la Figura 7, en donde se puede apreciar como variable espacial un rango altitudinal y cada área corresponde, no sólo a este gradiente, sino que, como mapa síntesis cualitativo, tiene asociadas unas características particulares mostradas gráficamente en el premapa de la Figura 6 , lo que pone en evidencia su utilidad, ya que facilita no sólo la lectura de la información espacial, sino también el paso al mapa, al permitir definir espacios que pueden ser caracterizados con mayor facilidad que con los datos crudos. 


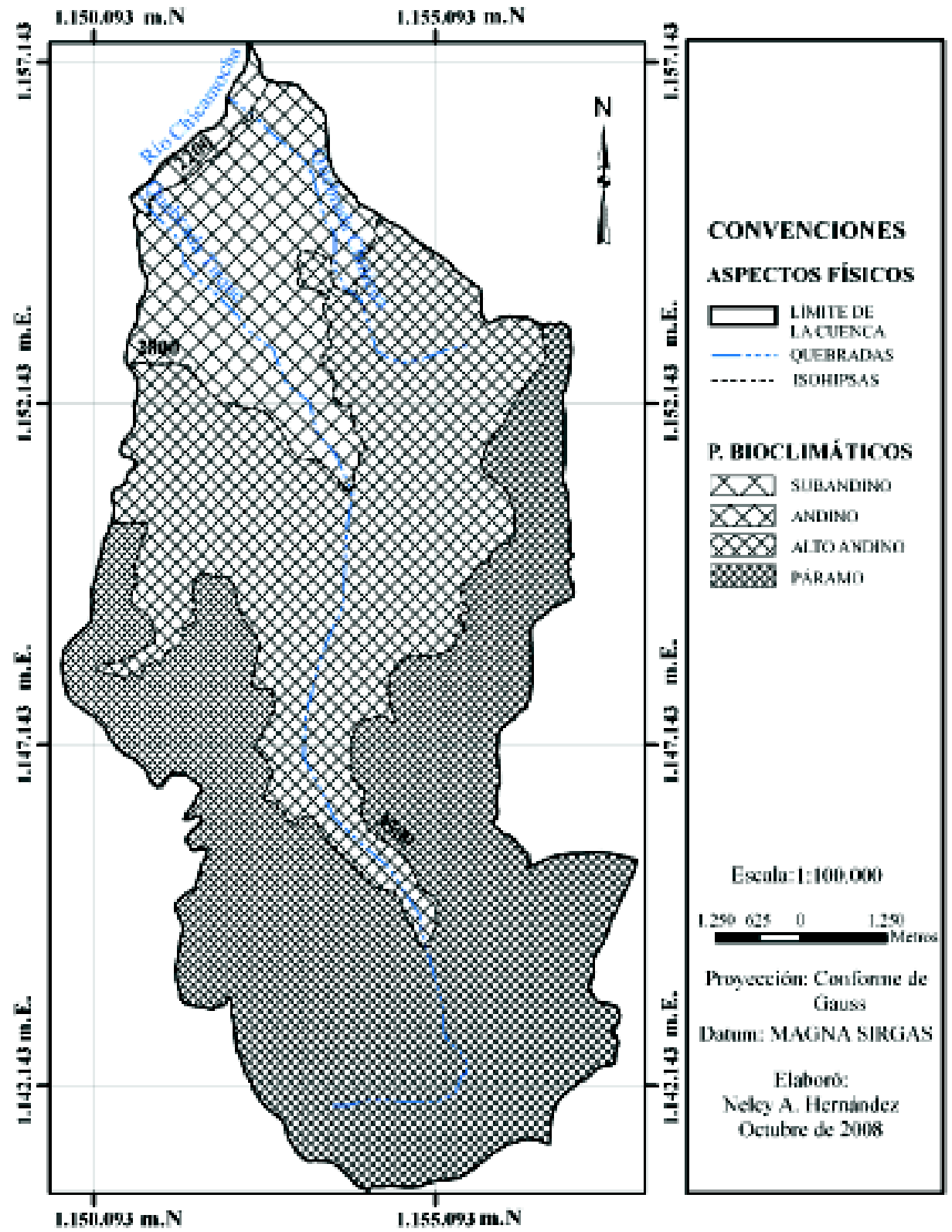

Figura 7. Pisos Bioclimáticos Potenciales en la Cuenca de las Quebradas Tirque y Chinicua. Municipio de Socha (Boyacá).

Fuente: Sáenz, 2003.

74 N. Hernández 
Así, no se desconoce la capacidad de la matriz geográfica o base de datos georreferenciada como premapa origen, y se verifica la propiedad de los premapas por mostrar distribuciones espaciales, que pueden ser cartografiadas.

Finalmente, es necesario aclarar que al hablar del premapa, en este caso el de la Figura 6, como reemplazo al mapa, aunque parece un poco pretencioso $\mathrm{y}$ excluyente, no significa la negación de éste como modelo gráfico útil en la representación de fenómenos geográficos, porque, si bien, el premapa puede ser presentado como resultado o como síntesis geográfica, el mapa base ha sido necesario en la delimitación de áreas y puede llegar a acompañarlo en determinados momentos, o configurarse como mapa síntesis, teniendo en cuenta que existe todo tipo de lector, desde el que tiene un nivel de referencia elemental, a quien únicamente puede interesarle el dónde ocurre un fenómeno, sin importar las relaciones que se den dentro del espacio estudiado, hasta aquel con un nivel avanzado que tenga la capacidad de ver las correlaciones entre variables, y no necesitar el mapa para ubicarlas.

Los planteamientos hechos aquí constituyen un llamado a la reflexión, sobre la importancia de utilizar los mapas para reconocer en ellos su poder analítico y sintético, evitar que sean sobrevalorados o subvalorados y perder su papel en la transmisión del conocimiento de las relaciones espaciales entre variables. Esto se logra pasando por el proceso que implica el análisis espacial con la ayuda de modelos gráficos, como los premapas, con los cuales no solamente se estudia la distribución de las variables, sino que, a partir de ellos, se selecciona la mejor forma de representación.

\section{Literatura citada}

Aguirre, C. 1979. Concepto interdisciplinario del análisis geográfico. En: Lurralde: Investigación y Espacio No. 2.

Bertin, J. 1977. La Graphique et le traitement graphique de l'information. París: Flammarion.

Board, C. 1967. Maps as models. En: Chorley y Haggett. (Eds). Models in geography. Great Britain. Methuen \& Co. Ltd.

Bolaños, J. 2004. Aplicaciones de la informática a la Geografía y Ciencias Sociales. En: Biblio 3W, Revista Bibliográfica de Geografía y Ciencias Sociales, Universidad de Barcelona, Vol. 9, No 503, 10 de abril de 2004. http://www.ub.es/geocrit/b3w-503.htm. Consultado en marzo de 2006. 
Bord, J. 1984. Initiation géo-graphique, ou comment visualiser son information. París: SEDES.

Bunge, William. 1967. Theoretical Geography. Lund Studies in Geography. Washington: C.W.K. Gleerup, Publishers.

Buzai, G. 2003. Mapas sociales urbanos. Buenos Aires: Lugar.

Caycedo, J. y Flórez, A. 1991. La información "Geo-gráfica". Los diagramas. En: Revista Cartográfica, 60: 39-87. México, D.F.: IPGH.

Chorley, R. y Haggett, P. 1967. Models in geography. Londres: Methuen \& Co Ltd.

Córdoba y Ordoñez, J. 2004. Geografía y Cartografía: reflexiones sobre el status científico de una simbiosis necesaria. En: Perspectiva Geográfica No. 10.

Durand, D. 1979. La systémique. Colección Que sais-je?. París : Presses Universitaires de France. Puf.

Ferras, R. 1993. Les Modèles graphiques en géographie. París: Economica/Reclus.

Fonsêca, A. 2004. Orientação geográfica: uma proposta metolológica para o ensino da geografia na $5^{\text {a }}$ série. Programa de pós-graduação e pesquisa em geografia. Natal. Universidade Federal do Rio Grande do Norte.

Harvey, D. 1983. Teorías, Leyes y Modelos en Geografía. Madrid: Alianza.

Hempel, C. 1965. La explicación científica. Estudios sobre la filosofía de la ciencia. Trad. 1979. Barcelona: Paidós Ibérica.

Hempel, C. 1988. Fundamentos de la formación de conceptos en ciencia empírica. Madrid: Alianza editorial.

IDEAM, Instituto de Hidrología, Meteorología y Estudios Ambientales. 2001. Atlas de Geomorfología. Bogotá. En: http://www.ideam.gov.co/atlas/mgeomorf.htm\#. Consultado Agosto de 2008 .

IGAC. Instituto Geográfico Agustín Codazzi. 1967. Atlas de Colombia. Bogotá.

IGAC. Instituto Geográfico Agustín Codazzi. 1969. Atlas de Colombia. Bogotá.

IGAC. Instituto Geográfico Agustín Codazzi. 1977. Atlas de Colombia. Bogotá.

IGAC. Instituto Geográfico Agustín Codazzi. 1992. Atlas de Colombia. Bogotá.

IGAC. Instituto Geográfico Agustín Codazzi. 2002. Atlas de Colombia. Bogotá.

\begin{tabular}{l|l}
\hline 76 & N. Hernández
\end{tabular} 
INGEOMINAS. 2005. Atlas Geológico de Colombia. Bogotá. En: http://www.ingeominas.gov.co/ content/view/761/316/lang,es/. Consultado Agosto de 2008.

Jeffers, J. 1991. Modelos en Ecología. Barcelona: Oikus-tau S.A.

Joly, F. 1982. La cartografía. Barcelona: Ariel Geografía.

Krugman, P. 1991. Increasing Returns and Economic Geography. En: Journal of Political Economy. pp.483-499.

Madrid A. y Ortiz L. 2005. Análisis y síntesis en geografía: algunos procedimientos. Bogotá, D.C: Unibiblos.

Minshull, R. 1975. An Introduction to Models in Geography. Londres: Longman Inc.

Moreno, A. 1994. Las clasificaciones. En: Bosque, J. (Ed.). Prácticas de análisis exploratorio y multivariante de datos. Barcelona: Oikus Tau.

Nagel, E. 1968. La estructura de la ciencia. Problemas de lógica de la investigación científica. Buenos Aires: Paidós.

Nagel, E. 1972. Simbolismo y Ciencia. En: Symbolism and Values. Editado por L. Bryson y otros, Ediciones nueva visión.

Richards, K. 2004. Exploring a typology for models in geography. En: Geography Online: Volume 5, Number 1. Spring 2005.

Rimbert, S. 1968. Leçons de cartographie thématique. París: Sedes.

Rodríguez, M. 2000. Modelos sociodemográficos: Atlas social de la Universidad de Alicante: Tesis de doctorado, Facultad de Ciencias Económicas.

Saenz, M. 2003. Análisis socio ambiental del uso del agua en la cuenca de las quebradas Tirque y Chinicua. Tesis no publicada. Bogotá, D.C.: Universidad Nacional de Colombia.

Santos, M. 1996. Metamorfosis del espacio habitado. Barcelona: Oikus-tau.

Vargas, E. 2004. Productos cartográficos temáticos enfocados a la representación de variables censales y del nivel demográfico. Tesis no publicada. Bogotá, D.C.: Universidad Distrital Francisco José de Caldas, Facultad de Ingeniería.

Zonneveld, I. 1988. The land unit. A fundamental concept in landscape ecology and its applications. ITC Report, The Netherlands, Enschede. 
Fecha de recepción: 15 de diciembre de 2008

Fecha de aprobación: 20 de diciembre de 2008 